could be regulated by a Gaede pump and measured by a McLeod gauge) a continuous discharge was maintained for about eight hours on several consecutive days, and I have observed a gradual hardening in spite of the maintenance of a comparatively high pressure. Further experiments carried out in this direction have revealed a remarkable effect which takes place in an X-ray bulb or, more generally, in any vacuum tube after a sufficiently long and continuous run--an effect similar to the polarisation of an electrolytic cell. This is, that after the discharge has been kept running for a sufficiently long time through a tube (inside which the pressure is kept nearly constant) a time arrives when the current flowing through the tube begins to decrease, and finally ceases altogether. To continue the discharge it is then necessary to increase either the potential difference applied to the electrodes or the pressure inside the tube. By repeating this operation several times I could ultimately reach a stage where a potential difference of more than 50,000 volts was not sufficient to produce a discharge in the bulb, although the pressure was as high as $0.060 \mathrm{~mm}$., whereas under ordinary conditions in the same bulb a much smaller potential difference was sufficient to produce a discharge under a pressure of the order of $0.00 \mathrm{Im}$. After the discharge has been stopped the bulb gradually returns to its normal condition, but afterwards a comparatively short run is sufficient to bring the bulb back to the state of polarisation.

It could be further shown that the effect is not due to changes in the nature of the gas in the bulb brought about by the discharge. A large side-tube containing two electrodes, the shape and distance apart of which were essentially the same as in the X-ray bulb, was fused into it. When after a long run the main bulb became polarised, so that the highest available voltage could not break down its resistance in spite of a high pressure of about $\mathrm{I} / 20 \mathrm{~mm}$., a potential difference of 1200 volts, supplied by a battery of small cells, when put across the side-tube was found to produce a normal discharge.

Experiments, which will be described elsewhere, give some evidence in support of the view that this effect is due to the destruction by the discharge of the gaseous layer on the surface of the electrodes.

It seems probable that the hardening of an X-ray bulb with usage is due not only to the disappearance of the gas in the bulb, but also to the phenomenon described in this letter. $S$. RATNER.

The Physical Laboratory, Victoria University, Manchester, June I.

\section{Observations of Plant-growth with the Recording Ultramicrometer.}

AT the meeting of the Royal Dublin Society on January 25 last, as reported in NATURE for February 24 , p. 850 , I described a form of ultramicrometer in which the minute movement of one plate of a parallel plate condenser, forming part of a thermionic-valve oscillating circuit, is recorded by a galvanometer. We are now applying this apparatus to the study of plant-growth, and as some of the preliminary observations show very clearly the pulsations of growth described by Sir J. C. Bose, it may be of interest to give a short account of these results.

In applying the method to this problem the upper plate of the condenser is a thin aluminium disc, about $6 \mathrm{~cm}$. in diameter, supported by a long flat strip of spring steel, so as to be situated about $\mathrm{r} / \mathrm{ro} \mathrm{mm}$. above the lower fixed (horizontal) plate. The latter plate may be given small vertical movements by means of a micrometer screw for adjustment or cali- bration of the apparatus. In many of our observations the apparatus is adjusted to give 150 divisions on the galvanometer scale for a displacement of the upper plate through $\mathrm{I} / \mathrm{I} 000 \mathrm{~cm}$., but it can be made many times more or less sensitive simply by altering the galvanometer shunt.

To the recording (upper) plate is rigidly attached a short wooden arm against which the plant member presses lightly. It is found that a weight of $\mathrm{I} / \mathrm{IO}$ gram placed on this causes a galvanometer deflection of roo divisions. This indicates the order of magnitude of the stress on the plant under observation. During experiments on roots the root-tip presses

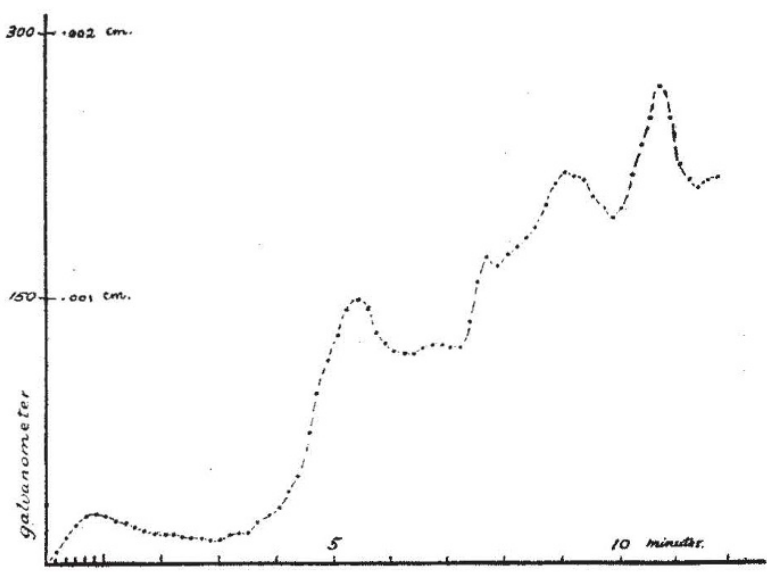

Fig. t. - Broad bean root-shoot (four days old),

the plate downwards; in other cases the movement is upwards.

As an example of one type of record obtained the accompanying curve is appended. It represents the (downward) growth of the root-shoot of a broadbean which had been planted some four days before and had just been removed from the ground. A considerable time had been allowed to elapse after placing the plant in position before observations were commenced.

I have to thank two botanical students, Miss Cannon and Mr. Saunders, for the part they are taking in the work. JOHN J. DowLing.

Department of Physics, University College, Dublin.

\section{Cup and Ring Markings.}

REFERRING to the note anent the above which appeared in NATURE of June 9 , p. 468 , may I mention that these peculiar surface-features can frequently be seen upon old mortar, stucco, and calcareous sandstones, and that they are due to molecular re-arrangement of the calcium carbonate, and not to any artistic efforts on the part of prehistoric man, as is frequently supposed?

At the Royal Society in 1896 I exhibited photographs of some remarkable examples of "cup and ring markings" which had developed on the stucco of one of the houses in Warrior Square, St. Leonardson-Sea. Similar "patterns " may sometimes be seen on old "American cloth" which has been subjected to tension, and also on old oil paintings. In these cases the gradual shrinkage of the canvas backing has produced the effect by causing lines of fracture in the more homogeneous layers of paint.

June II.

C. Carus-Wilson. 